\title{
„Geld- und Ordnungspolitik im Alten Reich“
}

\author{
MiCHAEL NORTH
}

Was Geld ist, war und sein wird, ändert sich im Laufe der geschichtlichen Entwicklung. Während das Geld in den modernen Volkswirtschaften nach seinen drei Grundfunktionen, des Tauschmittels, des Wertaufbewahrungsmittels und der Recheneinheit, definiert wird, besaß „Geld“ in anderen historischen Epochen eine unterschiedliche Bedeutung.

So fand das Geld beispielsweise in den wenig entwickelten Gesellschaften als Hortgeld oder als Repräsentations- und Sakralgeld zur Repräsentation und zur Wertanlage Verwendung und erst allmählich bildeten sich die modernen Geldfunktionen heraus. Welche Funktionen das Geld übernahm und welche „Stoffe“ (Nichtmetall - Metall - Papier) als Geld verwendet wurden, bestimmte der Entwicklungsstand der Geldwirtschaft, d.h. der Monetarisierungsgrad der Gesellschaft. ${ }^{1}$ Der Bewohner des neuzeitlichen Alten Reiches war in erster Linie mit Münzgeld konfrontiert, und allein die Kaufleute machten Gebrauch vom Buchgeld oder Kreditpapieren wie Wechsel oder Inhaberschuldschein. Charakteristisch war die Vielfalt der Münzprägung und damit der umlaufenden Münzen. Ursache dafür war die Aushöhlung der königlichen Münzhoheit im Hochmittelalter, als Bistümer und Abteien das Münzrecht oftmals zusammen mit Zoll- und Marktrecht erhielten. In der Folgezeit prägten die Herzöge neben dem König kraft Amtes, Bischöfe, Äbte und Grafen aufgrund neuer Privilegien, und mit den Städtegründungen kamen neue Münzberechtigte hinzu. Es verwundert daher nicht, dass in den Bemühungen zur Reichsreform seit dem ausgehenden 15. Jahrhundert auch ein einheitliches Währungssystem zur Debatte stand. Im Jahre 1524 schien dieses Wirklichkeit $\mathrm{zu}$ werden, als der Reichstag zu Esslingen eine erste Reichsmünzordnung verabschiedete, die den sächsischen Taler (Guldiner) zur Leitmünze und damit zur Grundlage des neuen Reichsmünzsystems machte. ${ }^{2}$ Da die meisten Münzstände die neue Ordnung nicht akzeptierten, ging die Diskussion um eine Reichsmünzordnung weiter. Auf dem Wormser Reichstag (1545) und den folgenden Reichsmünztagen, Kreistagen und Probationstagen wurden die Einführung einer Reichs(grob)münze, die Kleingeldversorgung sowie der Ein-

1 M. North, Kleine Geschichte des Geldes. Vom Mittelalter bis heute, München 2009, S. 7 f.

2 K. SCHNeIDER, Reichsmünzordnungen, in: M. NORTh (Hg.), Von Aktie bis Zoll. Ein historisches Lexikon des Geldes, München 1995, S. 336-338. 
und Abfluss fremder und einheimischer Münzen debattiert. Dazu verschaffte sich der Wormser Reichstag zunächst eine Expertise der silberfördernden Territorien (Münzstände), die dann in einer Empfehlung des Münzausschusses verarbeitet wurde und schließlich in ein vorläufiges Münzedikt mündete. Da Münzprägung immer ein Geschäft (manchmal auch ein Zuschussgeschäft) war, kollidierten unterschiedliche Interessen miteinander, die sich in einem globalen Umfeld - keine andere Ware fließt so schnell wie Münzen - behaupten mussten. Während die Städte an den gemeinen Nutzen des Reiches appellierten, stellten die Bergwerksbetreiber die Situation ihrer sich allmählich erschöpfenden Bergwerke in den Vordergrund. Dagegen versuchten Kaiser und Münzausschuss sowohl Interessen auszugleichen als auch eine schrittweise Vereinheitlichung, die dann auch im Reich und seinen Kreisen Wirklichkeit werden sollte. ${ }^{3}$

Auf dem Reichsmünztag zu Speyer (1557) wurden die Grundlagen für die Reichsmünzordnung von 1559 gelegt. ${ }^{4}$ Diese setzte mit dem Silbergulden eine neue Reichsmünze ebenso fest, wie andere Münzsorten, die künftig allein im Reich geprägt werden durften. In den Kreisen mussten Münzwardeine gewählt werden, die die umlaufenden Münzen ebenso wie die Münzstätten kontrollierten. Münzprobationstage analysierten die Mängel des Münzwesens auf der Kreisebene und sollten Maßnahmen dagegen beraten und ergreifen. Die Umsetzung der Reichsmünzordnung stieß zum Beispiel auf Widerstand, der sich im Niedersächsischen Kreis auch in einem „Bedenken“ an den Kaiser (1561) äußerte. ${ }^{5}$ Da man zunächst ohne Antwort blieb, verhandelten die wendischen Städte die Münzangelegenheiten weiterhin unter sich (,,da sie bis auf den negstkünftigen Reichstag sollten verschoben werden, noch eine lange Zeit also anstehen möchten“6). Als eine kaiserliche Antwort kam, bekräftigte diese die Einführung der Reichsmünzordnung in den Erblanden, blieb aber in bezug

3 R. Aulinger (Bearb.), Deutsche Reichstagsakten unter Kaiser Karl V., Bd. 16, Der Reichstag zu Worms 1545, Teilbd. 2, München 2003 (= Deutsche Reichstagsakten, Jüngere Reihe, Bd. 16,2), Nr. 66-89, S. 872-978. Einen guten Einblick in die Beteiligung einzelner Reichsstände am Diskussionsprozess bietet Joachim Krüger für die pommerschen Herzöge. J. KRÜGER, Zwischen Reich und Schweden. Die landesherrliche Münzprägung im Herzogtum Pommern und Schwedisch-Pommern in der Frühen Neuzeit (ca. 1580-1715), Münster, Berlin 2006 (= Nordische Geschichte, Bd. 3), S. 42-44, 53-56.

4 Deutsche Reichstagsakten, Reichsversammlungen 1556-1662. Der Kurfürstentag zu Frankfurt 1558 und der Reichstag zu Augsburg 1559, Dritter Teilband, bearb. v. Josef Leeb, Göttingen 1999, Nr. 804, S. 1953-1988. Im Weiteren folge ich meinem Aufsatz „Reich und Reichstag im 16. Jahrhundert“ in: M. LANZINNER und A. STROHMEYER (Hg.), Der Reichstag 1486-1613: Kommunikation - Wahrnehmung - Öffentlichkeiten (Schriftenreihe der Historischen Kommission bei der Bayrischen Akademie der Wissenschaften, Bd. 73), Göttingen 2006, S. 224-227.

5 M. v. BAHRFELD, Niedersächsisches Münzarchiv, Nr. 336-339, S. 267-282.

6 Ebd., Nr. 345, S. 287 f. 
auf das Münzbedenken „etwas dunkel“.7 1564 forderte der Kaiser die Einführung der Münzordnung im Niedersächsischen Kreis dann unnachgiebig ein. Dennoch vereinbarte der nächste Kreistag, die Münzberatungen des bevorstehenden Reichstages abzuwarten. ${ }^{8}$

Der Augsburger Reichabschied von 1566 mit seiner Änderung der Reichsmünzordnung von 1559 brachte dann den entscheidenden Wendepunkt für die Ausgestaltung der Münzverhältnisse im Niedersächsischen und Obersächsischen Kreis. Indem der alte Taler wieder zugelassen wurde (seine Prägung war 1559 zugunsten der Silberguldenprägung verboten worden), wuchs die Akzeptanz der Münzordnung in beiden Kreisen und gab die Basis für Münzordnungen der Kreise (1568 bzw. 1571).

Der Kreistag des Niedersächsischen Kreises bestätigte im Januar/Februar 1568 die Kreis-, Münz- und Probierordnung und wies alle Kreisstände an, diese einzuhalten. Zwei Kreis-Münzwardeine wurden bestellt, die die einzelnen Münzstätten bereisten und auf den zweimal jährlich stattfindenden Münzprobationstagen darüber zu berichten hatten. Wie alle Münzordnungen enthielten diese Bestimmungen zu Schrot und Korn der Münzen, Listen der zugelassenen Münzen, Regelungen zum Umtausch der nunmehr verbotenen Münzsorten sowie zur Prägung der Kleinmünzen. Auch wenn die Hansestädte sich noch zierten und vergeblich versuchten, ihre bisherige Sonderstellung innerhalb der Kreismünzordnung beizubehalten, hatte der Niedersächsische Kreis als einer der ersten die Umsetzung des Augsburger Abschiedes bzw. der Reichsmünzordnung von 1559 abgeschlossen. 1571 wurde dann die Zahl der Münzstätten entsprechend dem Reichsabschied von Speyer und dessen Bekräftigung im Frankfurter Reichsdeputationstag (1571) auf vier reduziert (Braunschweig, Lübeck, Magdeburg und Bremen), zu denen 1572 noch Hamburg und Rostock als Seehandelsstädte hinzukamen. Die Münzstätten der silberfördernden Stände blieben weiter zugelassen.

Für den Obersächsischen Kreis bedurfte es erst des Speyrer Reichstags (1570), um die Reichsmünzordnungen im Kreis zu übernehmen. ${ }^{9}$ Dabei hatten Münzstände sich ohnehin bereits im Rahmen der Reichsmünzordnungen bewegt, während sich Sachsen im Frühjahr 1571 stillschweigend auf den Boden der Reichsmünzordnung von 1559/1566 begab.

Der stetig steigende Silberpreis ließ die hochwertigen Reichstaler jedoch zum Spekulationsobjekt werden, so dass diese angesichts des Defizits in der deutschen Handelsbilanz gegenüber dem Osten und Südosten ebenso gerne

7 Ebd., Nr. 351, S. 290.

8 U. GitTel, Die Aktivitäten des Niedersächsischen Reichskreises in den Sektoren „Friedenssicherung“ und „Policey“ (1555-1682), Hannover 1996, S. 302 f.

9 Deutsche Reichstagsakten, Reichsversammlungen 1556-1662. Der Reichstag zu Speyer 1570. Zweiter Teilband: Akten und Abschied. bearb. v. M. LANZINNER, Göttingen 1988, \$120-151, S. 1242-1250. Vgl. auch M. LANZINNER, Friedenssicherung und politische Einheit des Reiches unter Kaiser Maximilian II. (1564-1576), Göttingen 1993, S. 381-393. 
wie die Gulden exportiert wurden. Mit dem Silberabfluss ins Ausland und der Knappheit grober Silbermünzen hing auch die allseits als Problem empfundene Vermehrung der Scheidemünzen zusammen. Da sich das Reich in den Münzordnungen von 1559 und 1566 nur auf die Talerprägung konzentrierte, die Ausprägung der leichten unterwertigen Scheide- oder Kleinmünzen aber den Territorien übertrug, wurden nur die rentablen groben Sorten nach dem Reichsfuß gemünzt, die kleinen und mittleren Münzsorten aber nach dem billigeren jeweiligen Landesmünzfuß. So heißt es in einem Memorandum des Jahres 1594: „Die fürnehmste Ursache aber, dahero jezig Zeit aller Unrath inn der müntz enttstehet, ist diese, das der gering schäzigen Sortten unnd Landtmüntzen dem Edict zuwider, allzuvil und überhäuffig gemacht, und dargegen die grobe guette sortten zerbrochen, zu Pfennigen und dergleichen gering schätzigen gattung vermünzt worden sein und noch immer vermünzt werden. " 10

Die Münzstände wussten nicht, wie sie mit dem knapper werdenden Prägemetall einen stetig zunehmenden Bedarf an Kleinmünzen befriedigen sollten. In dieser Situation brachte nur eine unterwertige Ausprägung der Kleinmünzen Gewinn und deckte zumindest die Prägekosten. Nachdem einmal ein Anfang mit der unterwertigen Prägung gemacht war, verschlechterte die Aussicht auf Gewinne das Kleingeld weiter. Mit der Zeit gingen fast alle Münzstände dazu über, stark kupferhaltige Münzen in Umlauf zu bringen. Falls die Prägung nicht offiziell in den zugelassenen Kreismünzstätten geschah, reaktivierte man stillgelegte Münzbetriebe oder richtete neue Stätten zur Münzprägung ein. Diese sog. Heckenmünzen wurden meist an Münzmeister verpachtet, die im Auftrag der Münzherren immer schlechteres Kleingeld produzierten und in Verkehr brachten. Bedingt durch den Aufschwung der Heckenmünzerei konkurrierte eine Vielzahl von Münzstätten um das rarer werdende Prägemetall. Dies verteuerte die Prägung und trug - da der Münzgewinn vorrangig war - zur weiteren Verschlechterung des Kleingeldes bei. ${ }^{11}$

Diese führte zu Beginn des Dreißigjährigen Kriegs zu den inflationären Schüben der Kipper- und Wipperzeit. Abgeleitet wird der Begriff Kipper vom niederdeutschen Kippen. Dies bezeichnet sowohl das Beschneiden der Münzen als auch das Auskippen, Aussuchen und Auswechseln von Geldstücken mit Hilfe einer Münzwaage („Geldkippe“), d.h. den Vorgang, der ebenfalls mit dem Wort Wippen umschrieben wurde. Vor allem Flugschriften verbreiteten die Bezeichnung Kipper und Wipper in Nord- und Ostdeutschland im Laufe der 1620er Jahre. So heißt es beispielsweise auf dem Titelblatt von „Ein füer nye Leidt":

10 H.-J. GERHARD, Ursachen und Folgen der Wandlungen im Währungssystem des Deutschen Reiches 1500-1625. Eine Studie zu den Hintergründen der sogenannten Preisrevolution, in: E. SCHREMmER (Hg.), Geld und Währung vom 16. Jahrhundert bis zur Gegenwart, Stuttgart 1993, 69-84, hier S. 75.

11 K. Weisenstein, Die Kipper- und Wipperzeit im Kurfürstentum Trier, Koblenz 1991, S. 16-24. 
„van den falschen/

grundtlosen/mineydigen/landt/brandt/

schandt/crütz/etich/Schwöffel/füer/

palaver unde in affgrundt verdarfften

landschelmischen falschken muentemestern/

kipperen/wipperen. . ${ }^{2}$

Indem er aus seiner Sammlung derartiger Flugschriften ausgiebig zitierte, hat dann im 19. Jahrhundert Gustav Freytag in seinen Bildern aus der deutschen Vergangenheit die Begriffe Kipper und Wipper dem deutschen Bildungsbürgertum nahe gebracht und damit erstmals eine Epoche der Geldgeschichte für eine breitere Leserschaft dargestellt. ${ }^{13}$

Die wirtschaftliche Entwicklung litt zunächst nicht unter der fortschreitenden Kleingeldverschlechterung. Zum einen blieb die Kaufkraft der groben Münzen erhalten, zum anderen vergrößerte die Verringerung und Vermehrung der Scheidemünzen die Geldmenge und verbesserte so die Bargeldversorgung. Eine regelrechte Blüte erlebte die Silberspekulation, insbesondere das „Aufwechseln“ grober Sorten gegen Kippergeld. Da der Geldhändler das Wechseln von Talern durch ein Aufgeld attraktiv machte, erhielt sein Kunde für einen Reichstaler scheinbar mehr Geld als dieser Wert war. Denn er konnte nicht sehen, dass das neue Geld, das er für den Reichstaler erhalten hatte, nur nominell mehr wert war und schnell seinen Wert verlor. Gewinne machten deshalb allein die Geldhändler oder die Münzherren selbst. ${ }^{14}$ Erst als auch silberfördernde Territorien wie Braunschweig-Wolfenbüttel, Sachsen und die Habsburger Lande zur Finanzierung von Krieg und Aufrüstung zu Beginn des Dreißigjährigen Krieges in großen Mengen geringwertige Kippermünzen in Umlauf brachten, überwog auch in diesen Staaten die Aussicht auf Gewinne die Bedenken oder gar die Berufung auf die Reichsmünzordnung.

Aber schon kurze Zeit später floss das Kippergeld zu einem erheblichen Teil über Steuern zurück in die staatlichen Kassen und zwang die Regierenden erneut zum Handeln. Daher wertete die Mehrheit der Staaten im Laufe des Jahres 1623 das Kippergeld drastisch ab.

In Sachsen beispielsweise wurden die Kippermünzen verrufen, und ihre Besitzer konnten sich allein den Metallwert von den Münzstätten in neuem

12 F. REDLICH, Die deutsche Inflation des frühen 17. Jahrhunderts in der zeitgenössischen Literatur. Die Kipper und Wipper, Köln 1972, S. 35.

13 G. Freytag, Der Dreißigjährige Krieg. Die Kipper und Wipper und die öffentliche Meinung, in: DeRs., Bilder aus der deutschen Vergangenheit, Bd. III, Leipzig 1898, 145-187; zum Folgenden M. NORTH, Kleine Geschichte des Geldes, S. 100-106 sowie Ders., „Unrath inn der müntz“: Das Geld im Alten Reich, in: S. WendeHORST, S. WestPhal, Lesebuch Altes Reich (Bibliothek Altes Reich, Bd. 1), München 2006, S. 183-188.

14 Zum spekulativen Geldhandel siehe beispielhaft K. SCHNEIDER, Frankfurt und die Kipper- und Wipperinflation der Jahre 1619-1623, Frankfurt a. M. 1990, S. 43-60. 
Geld auszahlen lassen. ${ }^{15}$ In den Habsburger Territorien setzte man die „Lange Münze" auf 13,3 Prozent ihres Nennwertes herab, während die bayrischen Münzen „nur“ um 75 Prozent abgewertet wurden, d.h. immerhin einen Viertel ihres Wertes behielten. ${ }^{16}$ Die Reichsstadt Frankfurt hatte bereits im Jahre 1622 um die Währungsstabilität gekämpft, die hier vor allem durch die $\mathrm{He}-$ ckenmünzen der umliegenden Herrschaften gefährdet wurde. Denn diese schmolzen, nachdem das Kippergeld kein Geschäft mehr war, die Kippermünzen ein und prägten daraus unterwertige Taler, die sie mit Hilfe regelrechter Vertriebsorganisationen in den Geldumlauf einschleusten. Gegen die Geldflut half es wenig, diese Münzen zu beschlagnahmen. Daher vereinbarte Frankfurt zur Wiederherstellung der Geldwertstabilität eine Gemeinschaftsprägung mit Kurmainz, Hessen-Darmstadt und Nassau-Saarbrücken und verrief die Pfennigprägungen der vergangenen Jahre ebenso wie alle anderen Scheidemünzen. ${ }^{17}$

Ein weiteres Problem stellten die Schulden dar, die zur Kipperzeit eingegangen worden waren und deren Rückzahlung nun fällig wurde. Sollten diese in altem oder in neuem Geld zurückgezahlt werden? In Sachsen verfocht man ähnlich wie bei der Wiederherstellung der Währungsstabilität die harte Linie und ordnete an, dass sämtliche Darlehen in neuer Münze zurückgezahlt werden mussten, egal ob sie vor oder während der Kipperzeit gewährt worden waren. Aufgrund des Protestes der Bevölkerung und des Einmarsches der Wallensteinischen Truppen nach Sachsen im folgenden Jahr blieb die praktische Wirkung des Schuldenmandats aber gering, da kaum Schulden zurückgezahlt wurden..$^{18}$ Differenzierter und gerechter verfuhren die Reichsstadt Frankfurt und die mit ihr in einer Währungsunion assoziierten Territorien. Lautete der Darlehensvertrag auf grobe Sorten und eine Rückzahlung „Stück für Stück“ (Münze für Münze), musste das Darlehen auch „Stück für Stück“ abgetragen werden. Hatte man aber die Schuldverschreibung in "Gulden“ ausgestellt, wurde für die Rückzahlung der Wert des Guldens zum Zeitpunkt des Vertragsabschlusses zugrunde gelegt. ${ }^{19}$ Damit hielten sich die Verluste von Gläubigern wie von Schuldnern in Grenzen. Falls es dennoch zum Streit zwischen den Parteien um die Rückzahlung von Darlehen kommen sollte, wurden vielerorts gütliche Vereinbarungen empfohlen, die einer Überbelastung der Gerichte vorbeugen sollten.

Welche Folgen hatte die Kipper- und Wipperzeit für die Bevölkerung? Von Geldhistorikern und Numismatikern wird in der Nachfolge Gustav Freytags immer wieder behauptet: „Die Folgen hatte zum größten Teil der

15 RedLich (wie Fn. 12), S. 55.

16 H. Chr. Altmann, Die Kipper- und Wipperinflation in Bayern, München 1976, S. 172.

17 SCHNEIDER (wie Fn. 14), S. 68.

18 ReDlich (wie Fn. 12), S. 57f.; erst im Jahr 1853 wurde das Mandat außer Kraft gesetzt.

19 SCHNEIDER (wie Fn. 14), S. 70. 
kleine Mann zu tragen, der zuerst zur Annahme der Kippermünzen per Verordnung gezwungen war und jetzt diese Münzen in einem verringerten Wert wieder zurückgeben musste" $;{ }^{20}$ und die zahlreichen Flugschriften scheinen ihnen Recht zu geben. Wenn wir aber bedenken, dass die meisten Flugschriften aus Nord-, Mittel- und Ostdeutschland stammten, scheint eine Differenzierung dringend notwendig. Als Beispiel einer für die Kipper- und Wipperzeit typischen Situation kann Frankfurt gelten, das auch geographisch zwischen dem stark von der Kippermünze heimgesuchten Sachsen und den rheinischen Territorien lag, die durch ihre Nähe zu den monetär stabilen Niederlanden nur wenig von den Auswirkungen der Kipperzeit spürten. In Frankfurt erreichte der Kurs des Reichstalers in Kippergeld 1622 mit 7 fl. (Gulden) seinen höchsten Kurs, wogegen er in Süddeutschland auf 10 fl., in Böhmen auf über $11 \mathrm{fl}$, in Kursachsen sogar auf $15 \mathrm{fl}$. anstieg. ${ }^{21}$ Mit der fortschreitenden Kleingeldentwertung stiegen die Preise, soweit sie in Kippergeld notiert wurden. Dagegen blieben die Preise, falls man in groben Sorten rechnete oder zahlte, verhältnismäßig konstant. Die Verschlechterung des Kleingeldes und der damit zusammenhängende Kursanstieg der groben Sorten riefen bei Lohnarbeitern und Gewerbetreibenden unterschiedliche Reaktionen hervor. Lohnarbeiter forderten eine Anhebung der in den Lohntaxen festgesetzten Löhne. Metzger, Bäcker, Fischer und Markthändler weigerten sich, ihre Waren gegen Kippermünzen zu verkaufen und verlangten als Bezahlung grobe Sorten. Da der Frankfurter Rat dies zu unterbinden suchte, forderten Bäcker und Metzger mit dem Argument, dass sie ihre Einkäufe mit grober Münze bezahlen müssten, eine Erhöhung des Backlohns bzw. der Fleischtaxen. ${ }^{22}$ Dass die Handwerker Bezahlung in gutem Geld verlangten, spricht für ein Vorhandensein dieser Sorten in Form von Reichstalern und Philippstalern (Taler der Spanischen Niederlande).

Diese Tatsache bestätigen auch die aus der Zeit des Dreißigjährigen Krieges besonders häufig überlieferten Münzschätze, die einen erheblichen Bestand an Philippstalern aufweisen. ${ }^{23}$ Das heißt, die über Generationen angehäuften Ersparnisse scheinen nicht so schnell zerronnen zu sein, wie es die Flugschriften und die ihnen folgende Literatur suggerieren. Vergleiche mit der Inflation von 1922/23 gehen also völlig fehl. Mindestens ebenso interessant ist aber die Tatsache, dass die Rückkehr zu stabilen Geldverhältnissen in Kriegszeiten gelang. Ermöglicht wurde dies durch neue Formen der Kriegsfinanzierung, nämlich einerseits die Ausweitung des internationalen Subsidiensystems - Frank-

20 Weisenstein (wie Fn. 11), S. 22.

21 SCHNEIDER (wie Fn. 14), S. 132.

22 SCHNeIDER (wie Fn. 14), S. 64f.

23 Ebd., S. 66; N. KLÜSENDORF, Der Schatz von Feldkrücken, Stadt Ulrichstein, Vogelsbergkreis, verborgen ab 1627, Marburg 1985; DERS., Der Münzschatz von Herborn zur Kipperzeit in der Grafschaft Nassau-Dillenburg, Marburg 1989, S. 77f., 160f.; W. HEß, Münzfunde aus Hessen als Spiegel des Geldumlaufs, in: Hessisches Jahrbuch für Landesgeschichte 25 (1975), S. 162. 
reich finanzierte Schweden, die spanischen Habsburger die österreichischen Verwandten - und andererseits die Finanzierung der Kriegshandlungen aus den besetzten Gebieten durch Steuern und Kontributionen nach der Devise „der Krieg ernährt den Krieg" (Wallenstein). ${ }^{24}$

Nach dem Dreißigjährigen Krieg war die Reichsmünzordnung des Jahres 1559 noch immer formal in Kraft, obwohl ihre Bestimmungen hinsichtlich des Münzfußes bei den Scheidemünzen wie bei den Grobmünzen ständig ausgehöhlt wurden. Aufgrund des steigenden Silberpreises prägten viele Münzstände wie die Kurpfalz, Österreich oder Brandenburg leichtere Talerteilstücke als von der Reichsmünzordnung vorgeschrieben. Neue Vereinbarungen waren also nötig. Daher einigten sich 1667 Brandenburg und Sachsen im Kloster Zinna darauf, von nun an 10 1/2 Taler aus der Mark Silber zu prägen. Dieser Zinnaer oder 10 1/2-Taler-Fuß löste den alten 9-Taler-Fuß der Reichsmünzordnung ab. Der Reichstaler der Reichsmünzordnung blieb als Recheneinheit bestehen, während man als Umlaufmünzen allein Sechstel-, Drittel- und Zweidrittel-Taler prägte. Insbesondere die „Zweidrittel“, die - weil sie dem Rechnungsgulden entsprachen - auch Gulden genannt wurden, sollten die dominierende Grobmünze der Zukunft werden. ${ }^{25}$ Zunächst mussten sich die Zinnaer Partner aber erst der Flut schlechter Münzen erwehren, die von den nicht nach Zinnaer Fuß prägenden Territorien in Verkehr gebracht wurden. Der Zinnaer Fuß war nicht mehr zu halten. Deshalb einigten sich Kursachsen, Kurbrandenburg und Braunschweig-Lüneburg, das sich 1668 dem Zinnaer Fuß angeschlossen hatte, 1690 in Leipzig auf einen leichteren Münzfuß, nämlich den 12-Taler-Fuß, und beendeten damit die sog. zweite oder kleine Kipperzeit. ${ }^{26}$

Nicht alle Territorien lernten aus den Erfahrungen der Kipperzeit. So verzichtete Österreich im 18. Jahrhundert während des Österreichischen Erbfolgekrieges und des Siebenjährigen Krieges darauf, die Kriegskosten mit der Münzprägung zu bestreiten. Hierbei wirkte einerseits die Kipperzeit noch nach; andererseits hatten sich die Habsburger Kaiser bei ihrer Wahl in den Wahlkapitulationen zur Erhaltung und Bewahrung der Reichsmünzordnungen

24 A. ERnstberger, Hans DeWitte, Finanzmann Wallensteins, Wiesbaden 1954, S. 194; F. REDLICH, The German Military Enterpriser and his Work Force, 2. Bde., Wiesbaden 1964; J. KuNISCH, Wallenstein als Kriegsunternehmer. Auf dem Weg zum absolutistischen Steuerstaat, in: U. SCHUlTZ (Hg.), Mit dem Zehnten fing es an. Eine Kulturgeschichte der Steuer, München 1986, S. 153-161.

25 W. SCHWINKOWSKI, Die Reichsmünzreformbestrebungen in den Jahren 1665-1670, in: VSWG 14 (1918), S. 77-83; T. ChristmanN, Das Bemühen von Kaiser und Reich um die Vereinheitlichung des Münzwesens, Berlin 1988, S. 105.

26 F. VON SCHRÖTTER, Das preußische Münzwesen im 18. J. Münzgeschichtlicher Teil, I, Berlin 1904, S. 72-78; ChristmanN (wie Fn. 25), S. 118-24. Im gleichen Jahr wurden im Torgauer Rezess für die Kleinmünzen leichtere - nach Nominalen gestaffelte - Münzfüße vereinbart. 
verpflichtet. Daher brachte Österreich die Kriegskosten sowohl durch Anleihen als auch durch die Emission von Banknoten auf.

Friedrich der Große dagegen hatte keine Scheu, die Münzprägung zur Aufbesserung der Staatsfinanzen, insbesondere zur Kriegsfinanzierung zu benutzen. Dazu verpachtete er die Münzstätten im eroberten Sachsen an den Großunternehmer Veitel Ephraim, der dort unterwertige sächsische und polnische Münzen prägte. Die hieraus fließenden Gewinne reichten aber zur Kriegsfinanzierung allein nicht aus, so dass 1758 alle sächsischen und preußischen Münzstätten an das Münzkonsortium Veitel Ephraim, Moses Isaak und Daniel Itzig verpachtet wurden. Die Pächter machten dann auch vor der Verschlechterung der preußischen Münzen nicht halt. Nach dem Frieden von Hubertusburg (1763) verspürte die preußische Bevölkerung denn auch ,weniger den Segen des Friedens als die Folgen des Krieges". Die Berliner, wie der Bäckermeister Heyde in seiner Chronik klagte, litten noch im Mai 1763 an dem „,böse[n]' Geld, [das] noch bis dato im Gange, wodurch die Lebens Mittel immer in höheren Preis steigen". ${ }^{27}$ Ein Währungsschnitt war notwendig, der vom König, gestützt auf ausländische Berater und Finanzfachleute, energisch in Angriff genommen wurde. 1764 kehrte man zum 14-Taler-Fuß zurück, zog die Kriegsmünzen ein und tauschte sie nach ihrem Metallwert in neues Geld um. Die Besitzer der Münzen verloren oftmals über 50 Prozent ihres Geldvermögens. Um 1770 waren die Umprägungen der Kriegsmünzen abgeschlossen und so die monetären Hindernisse eines Wirtschaftsaufschwungs beseitigt, der dann im Rahmen der Weltkonjunktur auch nicht auf sich warten ließ. ${ }^{28}$

Trotz dieser von Zeit zu Zeit wiederkehrenden inflationären Perioden waren die Reichskreise bei der Beaufsichtigung des Münzwesens verglichen mit anderen europäischen Ländern durchaus erfolgreich. So trugen sie durch überregionale Münzbündnisse dazu bei, dass trotz des zersplitterten Münzwesens im Heiligen Römischen Reich ein größeres Maß an monetärer Stabilität herrschte als im zentral regierten und einheitlichen Wirtschafts- und Währungsraum Frankreich. ${ }^{29}$

27 H. Schultz (Hg.), Der Roggenpreis und die Kriege des großen Königs. Chronik des Berliner Bäckermeisters Johann Friedrich Heyde, Berlin 1988, S. 98.

28 NORTH (wie Fn. 1), S. 125-129.

29 M. NORTH (Hg.), Deutsche Wirtschaftsgeschichte. Ein Jahrtausend im Überblick, München ${ }^{2} 2005$; Ders., Das Reich als Wirtschaftsraum, in: H. Schilling, W. Heun, J. GÖtZmanN (Hg.), Heiliges Römisches Reich Deutscher Nation 962-1806. Altes Reich und neue Staaten 1495-1806, Bd. II, Essays, 29. Ausstellung des Europarates in Berlin und Magdeburg, Dresden 2006, S. 159-170, hier S. 165-169. 
